\title{
Provably Unforgeable Signatures
}

\author{
Jurjen N.E. Bos* \\ David Chaum ${ }^{\dagger}$
}

\begin{abstract}
Very strong definitions of security for signature schemes have been proposed in the literarure. Constructions for such schemes have been proposed, but so far they have only been of theoretical interest and have been considered far too inefficient for practical use.

Here we present a new scheme that sacisfies these strongest definitions and uses essentially the same amount of computation and memory as the widely applied RSA scheme. The scheme is based on the well known RSA assumption.

Our signatures can be thought of as products resulting from a two-dimensional Lamport scheme, where one dimension consists of a list of public constants, and the other is the sequence of add primes.
\end{abstract}

\section{Introduction}

One of the greatest achievements of modern cryptography is the digital signature. A digital signature on a message is a special encryption of the message that can easily be verified by third parties. Signatures cannot be denied by the signer nor faisified by other parties.

This article introduces a new signature scheme that combines the strength of the strongest schemes with the efficiency of RSA.

Signing a message of 245 bits in our scheme is possible in roughly 910 multiplications, and verifying it costs about 152 multiplications. In comparison, RSA, using the ISO/IEC standard 9796 redundancy scheme, takes roughly 768 multiplications (or 610 using addition chains) for signing, and 3 (or optionally 17) for verification. RSA signatures are 512 bits long, while ours requires an additional message counter. Thus, 16 extra bits give a scheme that allows 65,536 signatures per public key.

A variation involving pre-computation, signs short messages (64 bits) in 33 multiplications (not counting precomputation) and verifies in 35 multiplications.

After the introduction, we discuss other signature schemes relevant to this work. We discuss the Lamport signature scheme, on which this signature scheme is based, in detail. Then, the new scheme is explained, and the possible choices for parameter values are shown.

\footnotetext{
"This article is adapted from the dissertation "Practical Privacy" of Jurjen N.E. Bos, writuen while he was at CWI (the Dutch nationally funded centre for Mathematics and Computer Science). He is currently affiliated with Irdeto (a pay TV company) in Hoofddorp, Netherlands.

${ }^{\dagger}$ David Chaum is affilialed boh with CWI and Digi Cash (innov ators in electronic money sysems).
} 


\section{Signature scheme}

An overview of signature schemes, comparing securities, can be found in the paper mentioned earlier [GMR88]. We use their notation. They define a signature scheme as consisting of the following components:

- A security parameter $k$, that defines the security of the system, and that may also influence performance figures such as the length of signatures, running times and so on.

- A message space $\mathbf{M}$, that defines on which messages the signature algorithm may be applied.

- A signature bound $b$, that defines the maximal number of signatures that can be generated without reinitialization. Typically, this value depends on $k$, but it can be infinite.

- A key generation algorithm $\mathrm{G}$, that allows a user to generate a pair of corresponding public and secret keys for signing. The secret key $S$ is used for generating a signature, while the public key $P$ is used to verify the signature.

- A signature algorithm $\sigma$, that produces a signature, given the secret key and the message to be signed.

- finally, a verification algorithm, that produces true or false on input of a signature and a public key. It ouputs true if and only if the signature is valid for the particular public key.

Some of these algorithms may be randomized, which means that they may use random numbers. Of course, $\mathrm{G}$ must be randomized, because different users must produce different signatures. The signing algorithm $\sigma$ is sometimes randomized, but this tends to produce larger signatures. The verification algorithm is usually not randomized.

A simple example of a signature scheme is a trapdoor one-way function $f$. The function $f$ is used for verification by comparing the function value of the signature with the message to be signed, and $\sigma$ is the trapdoor of $f$. The main problem with such a scheme is that random messages $f(x)$ can be signed by taking a random signature value $x$. A simple solution is to let $M$ be a sparse subset of a larger space, so that the probability that $f(x)$ is a valid message for random $x$ is low. An example of a sparse subset is the set of "meaningful" messages.

\section{Related work}

The notion "digital signanure" was introduced in [DH76]. This paper, which can be considered the foundation of modern cryptography, discusses the possibility of digital 
signatures and the use of a trapdoor one-way function to make them.

[RSA78] is the original article on the RSA scheme. It introduces the famous RSA trapdoor one-way function. This function is still widely in use and is applied frequently. A well-known weakness of RSA is that it is multiplicative: the product of two signaures is the signature of the product. This potential problem can be prevented as above by choosing an appropriate sparse message space.

Since then, an enormous number of signature schemes have been proposed [Rab77, MH78, Sha78, Rab79, Lie81, DLM82, GMY83, Den84, GMR84, OSS84, ElG85, OS85, FS86, BM88, GMR88, CA89, EGL89, EGM89, Mer89, Sch89, SQV89, BCDP90, Cha90, CR90, Hay90, CHP91], applied [Wil80, Cha82, Gol86, Bet88], and broken [Yuv79, Sha82, Tu84, BD85, EAKMM85, Ro091]. We will not discuss all these schemes here; we only discuss the ones that are interesting to compare with the new scheme.

The schemes [Rab79, GMY83, GMR84, GMR88] are steps towards a provably secure signature scheme. The scheme described in the last article is secure in a very strong way: it is "existentially unforgeable under an adaptive chosen-message attack" with probability smaller than $1 / Q(k)$ for every polynomial $Q$. This means that generating a new signature is polynomially hard if signatures on old messages are known, even if the old signatures are on messages chosen by the attacker.

The scheme in [GMR88] is based on factoring. While our scheme is based on the slightly stronger RSA assumption, it is much more efficient. The signature scheme of [GMR88] uses a large amount of memory for the signer, and quite a lot of computation. Our scheme uses no memory at all, except for a counter and the public values, and signing and verifying takes about as much computation as RSA does, depending on the parameters.

\section{The Lamport Scheme}

Our scheme can be thought of an optimization for both security and efficiency of [GMY83]. To explain the new system, we compare it to the earlier Lamport scheme (explained already in [DH76, page 650]). To make a signature in this scheme, the signer makes a secret list of $2 k$ random numbers

$$
A=a_{1,0}, a_{1,1}, a_{2,0}, a_{2,1}, \ldots, a_{k, 0}, a_{k, 1} \text {, }
$$

applies a one-way function $f$ to all elements, and publishes the result $B$ :

$$
\mathbf{B}=\left\{\begin{array}{l}
f\left(a_{1,0}\right), f\left(a_{2,0}\right), \ldots, f\left(a_{k, 0}\right) \\
f\left(a_{1,1}\right), f\left(a_{2,1}\right), \ldots, f\left(a_{k, 1}\right)
\end{array}\right.
$$

The signature consists of the numbers $a_{1, m_{1}}, a_{2, m_{2}}, \ldots, a_{k, m_{k}}$ from the list $\mathbf{A}$ (one from each "column"), where $m_{1}, m_{2}, \ldots, m_{k}$ are the bits of the message to be signed. The lists $\mathbf{A}$ and $\mathbf{B}$ cannot be used again. 
The properties of Lamport's scheme are easy to verify:

- Signing a message is only the publication of the proper elements of $\mathbf{A}$.

- To forge a signature, one needs to find certain values from the list $\mathbf{A}$. How hard this is, depends on the security of the one-way function $f$.

- If the values $\mathbf{A}$ are only used for one signature, new signatures cannot be made from old ones.

- Verification of a signature consists of applying the one-way function to the signature values, and comparing them to the public values determined by the signed message.

The new system uses the same idea, with three important differences. first, the list $\mathbf{B}$ is replaced by another list that can be used for all signatures. Second, the list $\mathbf{A}$ is constructed from two lists so that less memory is needed to define it. Third, the elements of $\mathbf{A}$ in the signature can be combined into a single number.

\section{A small optimization}

There is a trivial optimization of Lamport's scheme that reduces the number of public function values to almost half, that we could not find in the literature. This optimization is independent of the signature scheme as such. Basically, the signer signs by publishing a $k$-element subset of the $2 k$ secret numbers. Lamport's scheme chooses a particular set of subsets of the set of $2 k$ elements, as shown above. The necessary property of this set of subsets is that no subset includes another.

There are other sets of subsets with the property that no subsets includes another. A largest set of subsets with this property is the set of all $k$-element subsets (a wellknown result from lattice theory). For these sets, it is easy to see that no subset includes another.

For example, in Lamport's scheme, the list of 6 elements

$$
\mathbf{A}=a_{1,0}, a_{1,1}, a_{2,0}, a_{2,1}, a_{3,0}, a_{3,1}
$$

allows us to sign messages of 3 bits. If we renumber $\mathbf{A}$ as $a_{1}, a_{2}, a_{3}, a_{4}, a_{5}, a_{6}$, we get the set of 20 three-element subsets of $A$ :

$$
\begin{aligned}
& \left\{a_{1}, a_{2}, a_{3}\right\},\left\{a_{1}, a_{2}, a_{4}\right\},\left\{a_{1}, a_{2}, a_{5}\right\},\left\{a_{1}, a_{2}, a_{6}\right\},\left\{a_{1}, a_{3}, a_{4}\right\}, \\
& \left\{a_{1}, a_{3}, a_{5}\right\},\left\{a_{1}, a_{3}, a_{6}\right\},\left\{a_{1}, a_{4}, a_{5}\right\},\left\{a_{1}, a_{4}, a_{6}\right\},\left\{a_{1}, a_{5}, a_{6}\right\}, \\
& \left\{a_{2}, a_{3}, a_{4}\right\},\left\{a_{2}, a_{3}, a_{5}\right\},\left\{a_{2}, a_{3}, a_{6}\right\},\left\{a_{2}, a_{4}, a_{5}\right\},\left\{a_{2}, a_{4}, a_{6}\right\}, \\
& {\left[a_{2}, a_{5}, a_{6}\right\},\left\{a_{3}, a_{4}, a_{5}\right\},\left\{a_{3}, a_{4}, a_{6}\right\},\left\{a_{3}, a_{5}, a_{6}\right\},\left\{a_{4}, a_{5}, a_{6}\right\} ;}
\end{aligned}
$$

this allows us to sign one of 20 messages, which is equivalent to more than 4 bits.

In general, there are

$$
\left(\begin{array}{c}
2 k \\
k
\end{array}\right) \text {, or about } \frac{2^{2 k}}{\sqrt{k \pi}}
$$

$k$-element subsets, so that we can sign messages of about $2 k-\frac{1}{2} \log _{2}(k \pi)$ bits. The original Lamport scheme allowed messages of only $k$ bits, so that we get almost 
a doubling of the message size for the same size of the list $\mathbf{B}$. This simple improvement can also be used in our new signature scheme.

To encode a signature, a mapping needs to be defined between messages and these subsets:

$$
s(\text { message })=\text { subset } .
$$

The simplest mapping just enumerates messages (interpreted as numbers from 0 onwards) to sets (seen as binary strings that denote 1 for presence and 0 for absence) in order. Such a mapping is easily and efficiently computed by the algorithm shown in figure 1. The binomial coefficients do not need to be computed by repeated multiplication and division. The first binomial coefficient is always the same, so it can be precomputed, and the others can be computed by one multiplication and one division by small numbers using the properties:

$$
\left(\begin{array}{c}
t-1 \\
e
\end{array}\right)=\left(\begin{array}{l}
t \\
e
\end{array}\right) \cdot \frac{t-e}{t} \text { and }\left(\begin{array}{l}
t-1 \\
e-1
\end{array}\right)=\left(\begin{array}{l}
t \\
e
\end{array}\right) \cdot \frac{e}{t} .
$$

The algorithm outputs ones and zeros corresponding to the elements in the resulting set.

Note that the Lamport scheme uses another mapping that maps numbers onto $k$ element subsets, but that only a small number of these sets are used.

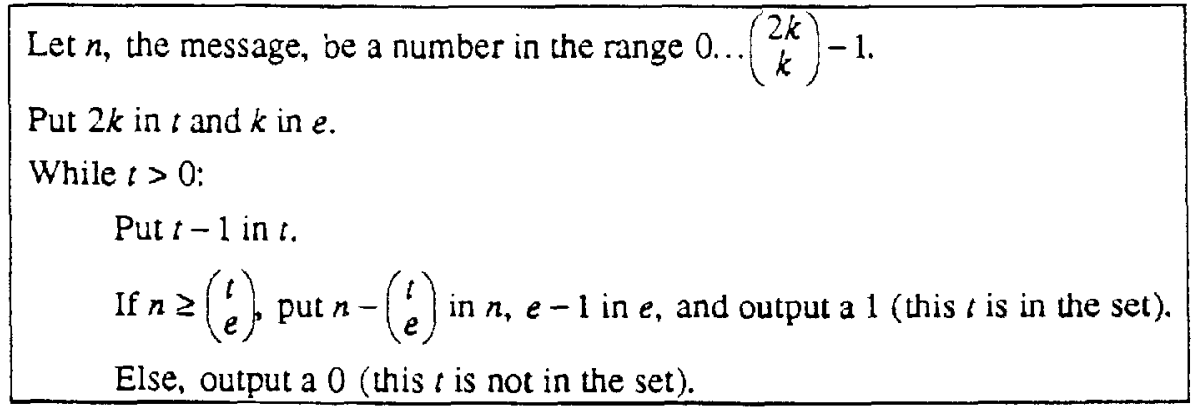

Fig 1. Algorithm for the mapping $s$.

\section{The New Signature Scheme}

The new signature scheme replaces the list $A$ of the Lamport scheme by a list of numbers that can be organized in a matrix. Instead of using a new list $B$ for every signature, a fixed list called $\mathbf{R}$ is used for all signatures and all participants. The one-way function $f$ is replaced by a set of trapdoor one-way functions, that changes per signature. For the trapdoor one-way functions, we use the modular root function of [RSA78].

The construction allows us to sign long messages using only a few numbers to define the set $\mathbf{A}$. In the example of figure 2, the set $\mathbf{A}$ of 12 elements is constructed 
from three primes $p_{1}, p_{2}, p_{3}$ (used only for this signature) and four public values $r_{1}, r_{2}, r_{3}, r_{4}$ (that can be used again). This set allows us to sign messages of 9 bits, since there are $924>2^{9}$ possible 6 -element subsets of $A$. Signing messages of 9 bits in the original Lamport scheme takes 18 public values that can be used only once.

\begin{tabular}{|llll|}
$\sqrt[p_{1}]{r_{1}}$ & $\sqrt[p]{r_{2}}$ & $\sqrt[p]{r_{3}}$ & $\sqrt[p]{r_{4}}$ \\
$\sqrt[p 2]{r_{1}}$ & $\sqrt[p 2]{r_{2}}$ & $\sqrt[p]{r_{3}}$ & $\sqrt[p 2]{r_{4}}$ \\
$\sqrt[p]{r_{1}}$ & $\sqrt[p 3]{r_{2}}$ & $\sqrt[p]{r_{3}}$ & $\sqrt[p 3]{r_{4}}$ \\
\hline
\end{tabular}

Fig. 2. Example list $A$ of the new scheme.

The numbers ai of $A$ are secret encryptions of the numbers $r i$ of $\mathbf{R}$, and the corresponding decryption exponents are public. The multiplicative property of RSA allows us to multiply the values of the signature to form one number. Verification of a signawure can be done using a simple computation, without having to compute the separate factors.

The public values of the new system are:

- One modulus per signer;

- The system-wide list $\mathbf{R}$. This list is used by all users, and that it does not change often, so that distribution does not require much traffic. The numbers in $\mathbf{R}$ are smaller than the smallest modulus used by the signers.

- A list of sets of primes that may be used for signing. For security reasons, the sets may not overlap each other, and the signers may only use these sets of primes.

A signature consists of the original message signed, the signature proper (an integer smaller than the modulus of the signer), and a description of the prime set.

In the language of [GMR88]:

- The security parameter determines the size of the RSA modulus. This modulus can vary per user.

- The message space $\mathbf{M}$ is (equivalent to) the set of subsets of $\mathbf{A}$ that include half the elements.

- The size of the public list of sets of primes determines the signature bound $b$.

- Key generation is a matter of generating an RSA modulus, and computing exponents for the modular root extractions.

- Signing and verification are defined below. 


\section{Signing}

For the list $\mathbf{A}$ of a signature, the set of RSA encryptions

is used, where:

$$
\mathbf{A}=\{\sqrt[R]{r} \bmod n \mid p \in \mathbf{P} ; r \in \mathbf{R}\}
$$

- P a set of primes from the public list;

- $\mathbf{R}$ is the public list of verification values;

- $n$ is the RSA modulus of the signer.

As explained above, a signature is constructed from a subset determined by $s(m)$ of half these numbers. The constant $k$ used in the algorithm that maps $s$ is equal to $\left\lfloor\frac{\# \mathbf{P} \# \mathbf{R}}{2}\right\rfloor$. This allows us to sign a message of almost \#A=\#P.\#R bits. The product of the elements of $A$ in this subset is the signature. Since this is a single number, the signature is much more compact than in Lamport's scheme.

Thus, signing a message consist of the following steps:

- Choose the set $\mathbf{P}$ of primes that is to be used for this signing from the public list. This determines $\mathbf{A}$ :

$$
\mathbf{A}=\left\{\sqrt[p_{i}]{r_{j}} \bmod n \mid i, j \in\{1, \ldots, \# \mathbf{P}\} \times\{1, \ldots \# \mathbf{R}\}\right\} .
$$

Like the sets $\mathbf{A}$ and $\mathbf{B}$ in Lamport's scheme, the set $\mathbf{P}$ can be used only once. The list A need not be computed.

- Determine the message $m$ to sign. This could be a message, or a public hash function value of that message, for example.

- Compute the subset $\mathbf{M}$ of index pairs from $(1, \ldots, \# \mathbf{P}\} \times\{1, \ldots, \# \mathbf{R}\}$ from the message $m$ with the algorithm described above:

$$
\mathbf{M}=s(m)
$$

- Compute the signature proper:

$$
S=\prod_{i, j \in \mathbb{M}} \sqrt[P_{1}]{r_{j}}(\bmod n)
$$

and send $m, \mathbf{P}$, and $S$ to the recipient.

There are two ways to increase the efficiency of signing. If there is time to do a precomputation, the entire set $A$ can be computed before the value of $m$ is known. Although this takes quite a while, signing becomes much faster, since signing consists only of multiplying the proper values of $\mathbf{A}$ together. If precomputation is not possible, the computation of $S$ can be speeded up with a vector addition chain [Bos92].

\section{Verification}

Instead of trying to compute individual factors of the signature, the number $S$ can be verified in a single computation. To see this, we note that the power of the signature 


$$
\prod_{S^{k \in P}} \rho_{\mathbf{k}}
$$

should be equal to the following product that can be computed from public values:

$$
\prod_{i, j \in M} \prod_{i \in M} p_{k} p_{k} / p_{j} .
$$

The lower product can be computed with a vector addition chain. Verification of a signature consists of checking that these two values are the same. The verification can be performed with a single vector addition chain, if the inverse of the signature is computed first:

$$
\left(S^{-1}\right)^{\prod_{k \in \mathbb{P}}} p_{k} \cdot \prod_{i, j \in \mathbf{M}} \prod_{r_{k} \in p^{\prime}} p_{k} / p_{j}
$$

which must evaluate to $1(\bmod n)$. To increase the efficiency of the verification, the signer could send $1 / S$ instead of $S$, so that the inversion is performed only once by the signer, and not by every verifier.

If not all prime numbers from $\mathbf{P}$ œccur as exponents in the set $\mathbf{M}$, it is possible to verify a signature using slightly fewer multiplications by raising $S$ to only the occurring primes. Unfortunately, this optimization is only applicable in the less interesting cases where verification requires a lot of multiplications.

The verifier must also check whether $P$ occurs in the public list. If $P$ is described as an index number in this list, this is of course unnecessary.

\section{Parameters}

In practice, the following parameter values could be used:

- A modulus size big enough to make factorization hard (200 digits, or 668 bits).

- R a list of 50 numbers.

- The sets $\mathbf{P}$ consisting of the $(5 n+1)^{\text {th }}$ to the $(5 n+5)^{\text {th }}$ odd prime number, where $n \in[0, \ldots, 16404\}$ is the sequence number of the signature. This uses the primes of up to 20 bits.

With these parameters, we have sets $A$ of 250 elements, so that a message of 245 bits ( 30 bytes) can be signed. A signature consists of the message, the signature product ( 668 bits, or 84 bytes), and the index number of the prime set ( 15 bits, or 2 bytes). Computing a signature takes about 1512 modular multiplications, and verification about 272; both these numbers are obtained using vector addition chains.

The list of the odd primes up to 20 bits (the highest being 1048557) can easily be stored; it would need only $64 \mathrm{~K}$ bytes of storage (using a bit table of the odd numbers) and contain 82025 primes. Such a list can easily be stored in a ROM chip. When all primes are used up, the user can choose a new modulus and start again. Another solu- 
tion is to change the list $\mathbf{R}$ often enough so that users do not run out of primes. To make it possible to verify old signatures, old values of $\mathbf{R}$ and the user moduli must be saved.

The list $\mathbf{R}$ can be computed from a seed number using a public hash function. This way, only one seed number is needed to define $\mathbf{R}$. This allows us one to use a long list $\mathbf{R}$ while using small amounts of data to distribute it. Also, less data is needed to save old lists.

Figure 3 shows the performance of the algorithm for several sizes of $\mathbf{R}$ and $\mathbf{P}$. For each of the entries in the table, the modulus is 668 bits (200 decimal digits), and the size of the primes in $\mathbf{P}$ is 20 bits. The entries are computed by averaging random number approximations. The entries marked by * have an estimated standard deviation higher than 10 , so that the last digits are likely to be inaccurate.

Powers and products were computed using addition chains and sequences; see [Bos92, chapter 4]. The products were computed collecting the base numbers; for example, the product

would be computed as

$$
b_{2}^{e_{1}} \cdot b_{3}^{e_{1}} \cdot b_{1}^{e_{2}} \cdot b_{3}^{e_{2}} \cdot b_{4}^{e_{2}} \cdot b_{2}^{e_{3}}
$$

$$
b_{1}^{e_{2}} \cdot b_{2}^{e_{1}+e_{3}} \cdot b_{3}^{e_{1}+e_{2}} \cdot b_{4}^{e_{2}}
$$

using a vector addition chain algorithm. In the cases were a single power was to be computed, the "window method" of [Bos92] was applied.

The table shows that in the general case, where verification is done more often than signing, it is advantageous to use a small $P$, possibly of only one element. The length of the list $\mathbf{R}$ is not a problem if it is generated from a seed, as suggested above. Another advantage of using a small set $\mathbf{P}$ is that the list $\mathbf{R}$ has $\boldsymbol{t}$ change less often.

\begin{tabular}{rr|rcc}
$\# \mathbf{R}$ & \# & message & sign & verify \\
\hline 250 & 1 & 245 & 910 & 152 \\
50 & 5 & 245 & 1512 & 272 \\
5 & 50 & 245 & 1451 & $2048^{*}$ \\
1 & 250 & 245 & 796 & $7123 *$ \\
500 & 1 & 495 & 1035 & 278 \\
50 & 10 & 495 & $2964 *$ & $1372 *$ \\
68 & 1 & 64 & 819 & 61 \\
17 & 4 & 64 & 1317 & 162 \\
4 & 17 & 64 & 1301 & $652^{*}$
\end{tabular}

Fig. 3. Performance for different size of $R$ and $P$.

The influence of the modulus size and prime size on the performance is shown is 
Figure 4. In this table, the size of $\mathbf{R}$ is set 1050 elements, while the sets $\mathbf{P}$ contain 5 elements each. The number of multiplications for signing depends on the size of the modulus only, while the number of multiplications for verifying depends on the size of the prime numbers only. Although it saves a little time during the signing to use a shorter modulus, we suggest using a modulus of 668 bits, since the current technology already allows factoring numbers of up to 351 bits.

The size of the primes in the sets $\mathbf{P}$ determines the verification time. Choosing smaller primes increases the speed of verification, but allows fewer signatures before a new list $\mathbf{R}$ is needed.

\begin{tabular}{c|cc|c} 
modulus size & signing & prime size & verifying \\
\hline 512 & 1172 & 10 & 171 \\
668 & 1512 & 20 & 272 \\
& & 30 & 381
\end{tabular}

Fig. 4. Performance for different sizes of modulus and primes.

If the elements of $A$ are precomputed, signing takes $\# A / 2-1$ multiplications. The precomputation takes about 796 \# A multiplications, so precomputation is only effective if there is plenty of time for doing it.

For extremely fast verification of signatures, we choose a list $\mathbf{R}$ of 68 elements, generated from a seed number that is part of the signature, and $\mathbf{P}=(3)$. For these parameters, the message to be signed is 64 bits (8 bytes). This allows verification of a signature in only 35 modular multiplications, plus the time to generate the elements of R. Signing takes about 819 multiplications. Using precomputation, signing takes 33 multiplications, but about 55000 multiplications for the precomputation.

\section{Proof of unforgeability}

We prove that the signature scheme is "existentially unforgeable under an adaptive chosen-message attack". This means that under the RSA assumption, if an attacker can influence the signer to sign any number of messages of his liking, he cannot forge new signatures in polynomial time, even if the messages depend on the signatures on earlier messages.

The main theorem used to prove unforgeability of the signature system is proved by Jan-Hendrik Evertse and Eugene van Heijst in [EH90], and is a generalization of a theorem by Adi Shamir [Sha83]. The theorem is about computing a product of RSA roots with a given modulus if a set of products of signatures is known. Under the RSA assumption, the theorem states that if a set of products of roots is known, the only new products of roots that can be constructed in polynomial time are those that can be 
computed using multiplication and division.

One assumption we make is that the attacker cannot combine the signatures of different participants, because they have different moduli. This is still an open problem. This assumption allows us to use the results of [EH90].

In our situation, we assume an attacker who knows many signature products $S$ from a participant. These products can be written as products of roots of elements of $\mathbf{R}$ :

$$
r_{1}^{x_{1}} r_{2}^{x_{2}} r_{3}^{x_{3}} \cdots r_{n R}^{x_{R} R}
$$

where the numbers $x_{i}$ are rational numbers. The theorem of [EH90] states that if we interpret the $x$ as vectors, the only new products that can be computed by the attacker correspond to linear combinations of these vectors. What remains to be proved is that linear combinations of these vectors do not give products that the attacker can use for new signatures.

The denominators of the rational numbers $x_{i}$ are products of primes from the set $\mathbf{P}$ of the corresponding signature, since the $x_{i}$ are sums of the form $\frac{1}{p_{1}}+\frac{1}{p_{2}}+\cdots$, where $p_{i} \in \mathbf{P}$. This means that we can speak of "the set of primes in a vector", meaning both the set of primes that occur in the denominators of the elements, and the set $\mathbf{P}$ used for generating the signature. Every signature uses another $\mathbf{P}$, and the sets $\mathbf{P}$ do not overlap, so the sets of primes in the vectors also do not overlap. A linear combination of vectors will contain only primes that occurred in the original vectors. From this we see that combining signatures with multiplication and division will not produce a signature with a set $\mathbf{P}$ that is not used before.

For a set $\mathbf{P}$ that has already been used, the only linear combination of vectors that contains the primes of $\mathbf{P}$ is a multiple of the corresponding vector, because any other linear combination of vectors contains primes not in $\mathbf{P}$. This means that other signature products do not help compute a new signature product with a given set $P$. From the definition of the signature product, we see that a power of a product cannot be a signature on another message, so this method also yields no new signatures for the attacker.

Note that if $m$ is a one-way hash function of a message, signatures on other messages can be forged if the hash function is broken. This is of course a separate problem from the security of the signature scheme.

From the above we conclude that an attacker cannot, under the RSA assumption, produce a signature product that is not already computed by the signer. This finishes the proof that the signature scheme is secure. 


\section{Conclusion}

It was already known that a signature with provable unforgeability existed under the factoring assumption. Our scheme, based on the modular root assumption, improves on the scheme in the literature on several points: signatures are smaller, while signing and verification use much less memory and computation. The new scheme has a large degree of flexibility, allowing the signing of both long and short messages by varying the parameters.

\section{References}

[BCDP90] J. F. Boyar, D. Chaum, I. B. Damgård and T. Pedersen: Convertible Undeniable Signatures, Advances in Cryptology: Proc. Crypto '90 (Santa Barbara, CA, August 1990), to be published.

[BD85] E. F. Brickell and J. M. DeLaurentis: An Attack on a Signature Scheme proposed by Okamoto and Shiraishi, Advances in Cryptology: Proc. Crypto '85 (Santa Barbara, CA, August 1985), pp. 28-32.

[Bet88] T. Beth: A fiat-Shamir-like Authentication Protocol for the ElGamal Scheme, Advances in Cryptology: Proc. Eurocrypt ' 88 (Davos, Switzerland. May 1988). pp. 77-86.

[BM88] M. Bellare and S. Micali: How to Sign Given any Trapdoor Function, Advances in Cryptology: Proc. Crypto '88 (Santa Barbara. CA, August 1988). pp. 200-215.

[Bos92] J. N. E. Bos: Practical Privacy, dissertation of the Eindhoven University of Technology. march 1992.

[CA89] D. Chaum and H. van Antwerpen: Undeniable Signatures, Advances in Cryptology: Proc. Crypto '89 (Santa Barbara, CA, August 1989), pp. 212-216.

[Cha82] D. Chaum: Blind Signatures for Untraceable Payments, Advances in Cryptology: Proc. Crypto '82 (Santa Barbara, CA, August 1982), pp. 199-203.

[Cha90] D. Chaum: Zero-knowledge Undeniable Signatures, Advances in Cryptology: Proc. Eurocrypt '90 (Ähus, Denmark, May 1990), pp. 458-464.

[CHP91] D. Chaum, E. van Heijst, and B. Pfitxmann: Cryptographically Strong Undeniable Signatures, Unconditionally Secure for the Signer, Advances of Cryptology: Proc. Crypto '91 (Santa Barbara, August 1991), to be published.

[CR90] D. Chaum and S. Roijakkers: Unconditionally Secure Digital Signatures, Advances in Cryptology: Proc. Crypto ' 90 (Santa Barbara, CA, August 1990), pp. 209-217.

[Den84] D. E. R. Denning: Digital Signatures with RSA and Other Public-Key Cryptosystems, Comm. ACM 27 (No. 4, April 1984), pp. 388-392.

[DH76] W. Diffie and M. E. Hellman: New Directions in Cryptography, IEEE Trans. Information Theory IT-22 (No. 6. November 1976), pp. 644-654.

[DLM82] R. DeMillo. N. Lyacb, and M. Merritt: Cryptographic Protocols, Proc. 14th ACM Symp. [EAKMM85] Theory of Computing (San Fransisco, CA, May 1982), pp. 383-400.

D. Estes, L. M. Adleman, K. Kompella, K. McCuriey, and G. L. Miller: Breaking the Ong-Schnorr-Shamir Signature Scheme for Quadratic Number fields, Advances in Cryptology: Proc. Crypto '85 (Santa Barbara, CA, August 1985), pp. 3-13. 
[EGL89] S. Even, O. Goldreich, and A. Lempel: A Randomized Protocol for Signing Contracts, Advances in Cryptology: Proc. Crypto '89 (Santa Barbara, CA, August 1989), pp. 205-210.

[EGM89] S. Even, O. Goldreich, and S. Micali: On-line/Off-line Digital Signatures, Advances in Cryptology: Proc. Crypto '89 (Santa Barbara, CA, August 1989), pp. 263-275

[EH90] J-H. Evertse and E. van Heyst: Which RSA Signatures can be Compused from Some Given Signarures?, Advances in Cryptology: Proc. Eurocrypt ' 90 (Arhus, Denmark, May 1990), pp. 83-97.

[EH91] J.H. Evertse and E. van Heyst: Which RSA Signarures can be Compused from Certain Given Signarures?, Repor W 91-06, February 1991, Marhematical Institute. University of Leiden.

[E]G85] T. ElGamal: A Public Key Cryptosystem and a Signature Scheme Based on Discrete Logarithm, IEEE Trans. Information Theory IT-31 (No. 4, July 1985), pp. 469-472.

[FS86] A. flat and A. Shamir: How to Prove Yourself: Practical Solutions of ldentification and Signarure Problems, Advances in Cryptology: Proc. Crypto '86, (Santa Barbara, CA, August 1986), pp. 186-194.

[GMR84] S. Goldwasser, S. Micali, and R. L. Rivest: A "Paradoxical" Solusion to the Signasure Problem, Proc. 25th IEEE Symp. Foundarions of Computer Science (Singer Island, 1984), pp. $441-448$.

[GMR88] S. Goldwasser, S. Micali, and R. L. Rivest: A Digital Signature Scheme Secure Against Adaptive Chosen-Message Attacks, SIAM Joumal on Computing 17 (No 2, April 1988), pp. 281-308.

[GMY83] S. Goldwasser, S. Micali, and A. Yao: Strong Signcrure Schemes, Proc. 15th ACM Symp. Theory of Computing (Boston, MA, April 1983), pp. 431-439.

[Col86] O. Goldrcich: Two Remarks Concerning the Goldwasser-Micali-Rivest Signasure Scheme, Advances in Cryptology: Proc. Crypto '86 (Santa Barbara, CA, August 1986), pp. 104-110.

[Gol86a] O. Goldrcich: Two Remarks Concerning the Goldwasser-Micali-Rivest Signature Scheme. Repon MIT/LCS/TM-315, Massachusetts Institute of Technology.

[Hay90] B. Hayes: Anonymous One-Time Signasures and Rexible Untraceable Electronic Cash, Advances in Cryptology: Proc. Auscrypt ' 90 (Sydney, Australia, January 1990), pp. 294305.

[Lie81] K. Lieberherr: Uniform Complexity and Digital Signatures. Theoretical Computer Science 16 (1981), pp. 99-110.

[Mau91] U. Maurer: Non-interactive Public Key Cryptography, Advances in Cryptology: Proc. Eurocrypt' 91 (Brighton, Uniled Kingdom, April 1991), to be published.

[Mer89] R. C. Merkle: A Certified Digital Signature, Advances in Cryptology: Proc. Crypto '89 (Santa Barbara, CA, August 1989). pp. 218-238.

[MH78] R. C. Merkle and M. E. Hellman: Hiding Information and Signatures in Trapdoor Knapsacks, IEEE Trans. Information Theory IT.24 (No. 5, September 1987), pp. 525-530.

[Oka88] T. Okamoto: A Digital Mulsisignarure Scheme Using Bijective Public.Key Cryptosystems, ACM Trans. Computer Systems 6 (No. 8, November 1988), pp. 342.441.

[OS85] T. Okamato and A. Shiraishi: A Fast Signatwe Scheme Based on Quadratic Inequalities, Proc. 1985 Symp. Security and Privacy (Oakland, CA, April 1985), pp. 123-132

[OSS84] H. Ong. C. P. Schnorr, and A. Shamir: Efficiens Signarure Schemes based on Polynomial Equarions, Advances in Cryptology: Proc. Crypto "84 (Santa Barbara, August 1984). pp. $37-46$.

[Rab77) M. O. Rabin: Digitalized Signatures, Foundations of Secure Computations 1977 (Atlanta, GA, October 1977), pp. 155-168.

[Rab79] M. O. Rabin: Digitalized Signatures and Public-key Function as Intractable as Factorization, Report MIT/LCS/TR-212, Massachusetts Institute of Technology. 
[Roo91] P. J. N. de Rooij: On the security of the Schnorr Scheme using Preprocessing. Proc. Eurocrypt ' 91 (Brighton, United Kingdom), to be published.

[RSA78] R. L. Rivest, A. Shamir, and M. Adleman: A Method for Obtaining Digital Signatures and Public Key Cryptosystems, Comm. ACM 21 (No 2, February 1978), pp. 120-126.

[Sch89] C. P. Schnorr: Efficient ldentification and Signatures for Smart Cards, Advances in Cryptology: Proc. Crypto '89 (Santa Barbara, CA, August 1989), pp. 239-251.

[Sha78] A. Shamir: A Fast Signature Scheme, Report MIT/LCS/TR-107, Massachuseus Institute of Technology.

[Sha82] A. Shamir: A polynomial Time Algorithm for Breaking the Basic Merkle-Hellman Crypiosystem, Proc. 23rd IEEE Symp. Foundations of Computer Science (Chicago, IL. 1982), pp. 145-152.

[Sha83] A. Shamir: On the Generation of Cryptographically Strong Pseudorandom Sequences. ACM Trans. Computer Systems 1 (No. 1, February 1983), pp. 38-44.

[Sha84] A. Shamir: /dentity-based Cryptosystems and Signature Schemes, Advances in Cryptology: Proc. Crypto '84 (Santa Barbara, CA, August 1984), pp. 47-53.

[SQV89] M. de Soete, J.-J. Quisquater, and K. Vledder: A Signature with Shared Verification Scherne, Advances in Cryptology: Proc. Crypto '89 (Santa Barbara, CA, August 1989), pp. 253-262

[Tu84] Y. Tulpan: Fast Cryptoanalysis of a Fast Signatwe System, Master's thesis in Applied Mathematics, Weizmann Instiwte, Israel, 1984.

[Wil80] H. C. Williams, A Modification of the RSA Public-Key Encryption Procedure, IEEE Trans. Information Theory TT.26, (No.6, November 1980), pp. 726-729.

[Yuv79] G. Yuval: How to Swindle Rabin, Cryptologia 3 (No. 3, July 1979), pp. 137-189. 